\title{
DNA analysis as a tool for breast cancer malpractice determination: An interdisciplinary approach
}

\author{
ERSI ABACI KALFOGLOU ${ }^{1}$, REHAT FAIKOGLU ${ }^{2}$, SEBNEM ÖZCAN ${ }^{1}$, GABRIEL PETRIDIS ${ }^{1}$, \\ HÜLYA YÜKSELOGLU ${ }^{1}$ and SEVIL ATASOY ${ }^{1}$ \\ ${ }^{1}$ Institute of Forensic Sciences, Istanbul University, 34303 Cerrahpasa, Istanbul; \\ ${ }^{2}$ Kirklareli College of Health, Trakya University, Kirklareli, Turkey
}

Received January 26, 2006; Accepted March 9, 2006

\begin{abstract}
Malpractice in breast cancer can be seen as falsenegative or false-positive findings which may result in either late or incorrect therapies. Biopsy material can be unintentionally interchanged, leading to incorrect treatment, and psychological damage to the patient. There is an obvious need for individualization of the tissue samples in such cases. In this study we used a multidisciplinary approach to integrate DNA technology that has been standardized and used in forensic science for other purposes, mainly to prove malpractice that has been the result of interchanging tissue samples in breast cancer. The main focus of the study was to evaluate the applicability of the technique, therefore we studied the samples of a 58-year-old female for whom the result of pathological analysis was reported as 'invasive ductal carcinoma'. The patient was surgically treated by a modified mastectomy technique and referred for chemotherapy. Prior to chemotherapy we found that the tissue samples analyzed did not belong to the patient in question. We used a battery of 15 polymorphic STR loci to identify the sample and we had strong evidence for exclusion of the patient. The analysis was done on both blood and buccal swab of the patient and on the tissue sample. We concluded that the technique is applicable and useful; however care should be taken in the interpretation of the results because the mutations in the tumoral tissues are very well known. Therefore, the maximum of informative loci should be studied and loss of heterozygosity should always be considered. We should also have in mind the possibility of intentional interchange which gives the results value in medico-legal investigations.
\end{abstract}

\section{Introduction}

Breast cancer is an important public health problem in Turkey. The incidence of this disease and the resulting deaths

Correspondence to: Dr Ersi Abacı Kalfoglou, Institute of Forensic Sciences, Istanbul University, 34303 Cerrahpasa, Istanbul, Turkey E-mail: ersi@istanbul.edu.tr

Key words: breast cancer, malpractice, STR, DNA identification continue to increase, as 30,000 new cases of breast cancer were diagnosed last year in the country. Breast cancer forms approximately $30 \%$ of all cancers identified in women, and the incidence rate is twice as high as that for colorectal and lung/bronchus cancer (1). The international improvements in the detection and treatment of breast cancer have resulted in an increase in the long-term survival of patients. Consequently, the increase in breast cancer incidence ultimately results in a growing demand for supportive, palliative, and medical services (2). While developments in these sections are positive, there is a series of problems that can be classified as malpractices concerning the diagnosis, treatment and follow-up of the patients. One is the failure to see a cancer that is visible retrospectively (3). Screening mammography may miss a breast cancer thus presumably delay its diagnosis and treatment. It is reported that almost $25 \%$ of screening misses small tumors (4). False-negative rates of mammography are seriously criticized by numerous articles and a huge platform of discussion on issues concerning the benefits and limitations of the technique has been created $(5,6)$. Another problematic situation is the false-positive outcome that may have severe consequences for the patient resulting to unnecessary operations, chemotherapies and suffer from psychological damage. A false-positive result may be due to an unintentional interchange of biopsy material (7).

This study was carried out to show that the proof of such an interchange can be done by the analysis of the polymorphic DNA loci that are used in Forensic Science for personal identification. We also discuss the necessary precautions that should be taken in order to avoid later ubiquities. As an example we describe a patient who had an unnecessary operation because of such a mistake.

\section{Patients and methods}

The patient (58-year-old female) was admitted to a state hospital for evaluation of a mass in her right breast discovered during a physical examination and the specimen obtained by needle biopsy was evaluated as 'benign findings'. An excisional biopsy was obtained at the hospital and the result of pathological analysis was reported as 'invasive ductal carcinoma'. Hence, the patient was surgically treated by a modified mastectomy technique in the state hospital and referred for chemotherapy to Cerrahpasa Medical Faculty of Istanbul 
University. The pathology department of the Medical Faculty demanded a sample of the tumor from the pathology laboratory of the private clinic where the first biopsy was taken, for evaluation of tumor receptors. The study of the specimen failed to reveal the presence of any tumor tissue. Then slides and tissue blocks of the specimen removed at the state hospital was asked for examination. Perusal of these, verified presence of ductal carcinoma. However, the discrepancy among the macroscopic physical aspects of the slides and the blocks sent from the state hospital aroused suspicion concerning the origin of the tissue from the state hospital: possibly it was from another patient. The possibility of interchange of the specimens brought about the need for identification using DNA analysis and the tissues were sent to the Institute of Forensic Sciences of Istanbul University.

The samples used were paraffin blocks that were questioned together with blood and buccal swabs from the patient. We specifically wanted to examine the buccal swab to avoid the possibility of circulating mutated DNA. DNA from the blood and buccal swab samples was extracted using Qiagen DNA extraction kit. For the paraffin-embedded tissue we first applied deparaffinization and then we extracted DNA by the use of the same extraction kit. The DNA amount obtained was at the recommended concentration of $0.05-0.125 \mathrm{ng} / \mu 1$. The polymorphic loci that were amplified and their chromosomal locations are as follows: D8S1179 (8), D21S11 (21q11.2-q21), D7S820 (7q11.21-22), CSF1PO (5q33.3-34), D3S1358 (3p), TH01 (11p15.5), D13S317 (13q22-31), D16S539 (16q24-qter), D2S1338 (2q35-37.1), D19S433 (19q12-13.1), vWA (12p12pter), TPOX (2p23-2per), D18S51 (18q21.3), D5S818 (5q2131), FGA (4q28), Amelogenin (X: p22.1-22.3, Y: p11.2). For the fragment analysis the AmpFlSTR ${ }^{\circledR}$ Identifiler $^{\mathrm{TM}}$ PCR amplification kit with a five-dye fluorescent system for automated DNA fragment analysis was used. Following the manufacturer's instructions $10.5 \mu \mathrm{l}$ AmpFISTR PCR reaction mix, $5.5 \mu 1$ AmpFlSTR primer mix, $0.5 \mu 1$ AmpFISTR Taq Gold DNA Polymerase, $10 \mu 1$ DNA were used. The PCR parameters were as follows: incubation at $95^{\circ} \mathrm{C}$ for $11 \mathrm{~min}$; denaturation at $94^{\circ} \mathrm{C}$ for $1 \mathrm{~min}$, annealing and extension at $59^{\circ} \mathrm{C}$ for $1 \mathrm{~min}$, at $72^{\circ} \mathrm{C}$ for $1 \mathrm{~min}$ (for 28 cycles) final extension at $60^{\circ} \mathrm{C}$ for $60 \mathrm{~min}$. The products were analyzed on the ABI PRISM 310 Genetic analyzer using Data Collection software v2.1 and GeneScan analysis.

\section{Results}

The results of the polymorphic STR loci that were amplified can be seen in Table I. The samples studied were the blood and the buccal swab of the patient and the tissue sample that was under suspicion. There is a complete match in the blood and buccal swab samples D8S1179, D3S1358 and D19S433 loci were not identified in the tissue sample and there is exclusion in 11 loci mainly D21S11, D7S820, CSF1PO, THO1, D13S3317, D16S539 TPOX, D18S51, D5S818 vWA and FGA.

\section{Discussion}

The rapid growth of DNA-based tests raises complex questions on how to integrate them efficiently into clinical medicine (8). In this study we used a multidisciplinary
Table I. The STR profiles of the blood and buccal swab of the patient together with the profile of the tissue sample that was shown to belong to another person.

\begin{tabular}{lccc}
\hline Name of STR & Tissue & Blood & Buccal swab \\
\hline D8S1179 & & $14-15$ & $14-15$ \\
D21S11 & $31.2-32.2$ & $30-30.2$ & $30-30.2$ \\
D7S820 & $9-11$ & $8-9$ & $8-9$ \\
CSF1PO & $10-11$ & $13-14$ & $13-14$ \\
D3S1358 & & $17-18$ & $17-18$ \\
THO1 & $5-6$ & $7-8$ & $7-8$ \\
D13S3317 & $8-11$ & $11-11$ & $11-11$ \\
D16S539 & $8-12$ & $10-12$ & $10-12$ \\
D2S1338 & $18-19$ & $18-19$ & $18-19$ \\
D19S433 & & $13-14$ & $13-14$ \\
vWA & $14-16$ & $14-15$ & $14-15$ \\
TPOX & $8-12$ & $8-11$ & $8-11$ \\
D18S51 & $13.2-14.2$ & $14-14$ & $14-14$ \\
AMELOGENIN & $\mathrm{XX}$ & $\mathrm{XX}$ & $\mathrm{XX}$ \\
D5S818 & $11-13$ & $12-12$ & $12-12$ \\
FGA & $22-24$ & $23-24$ & $23-24$ \\
\hline
\end{tabular}

approach to integrate DNA technology that has been standardized and used in forensic science to prove malpractice that has been the result of interchanging tissue samples in breast cancer. The approach was to use polymorphic short tandem repeats (STRs) for identifying a suspicious tissue sample. The AmpFISTR ${ }^{\circledR}$ Identifiler ${ }^{\mathrm{TM}}$ PCR Amplification kit that is used in this study is a short tandem repeat (STR) multiplex assay that amplifies 15 tetranucleotide repeat loci and the Amelogenin gender determining marker in a single PCR amplification (9). All thirteen of the required loci for the Combined DNA Index System (CODIS) are included in this kit for evaluation of known-offender data in the United States. Two additional loci, D2S1338 and D19S433, are included. The combination of the 15 loci is consistent with several worldwide database recommendations (10). This ensures a practically positive identification of any unknown sample, taken that there is reference material to compare. This STR assay is widely used in forensic case work for human identification and paternity confirmation. The high power of discrimination make them useful clinical tools for following chimerism in allogenic marrow transplantation or in cases of suspicious diagnosis in paraffin-embedded tissues (11-13).

We examined the maximum possible STR loci (15+ Amelogenin) to obtain the required correct exclusion. The results can be seen in Table I. Three out of 16 loci were not clearly typed in the tissue sample. When comparing the tissue samples, the buccal swabs and the blood sample studied, the blood and the buccal swab show completely the same profile, whereas 11 out of 15 loci studied are different between the blood/swab and tissue. No homozygosity was observed in any of the loci in the malignant tissue sample, indicating that 
there is no allelic loss. It can be clearly seen that the two samples originated from different subjects.

Microsatellite alteration has been reported in many studies characterized by expansion or deletion of a repeat in malignant tissue. Microsatellite alteration or instability (MI) is possible and characterized by expansion or deletion of a repeat in malignant tissue and decrease in heterozygosity. It has been detected in breast cancer, in neurodegenerative diseases, in human atherosclerotic plaques, in spontaneously aborted embryonic tissues, in human pterygia and pulmonary sarcoidosis (14-18). There are numerous studies using this instability and high mutation rate of microsatellites to either try to explain or find clues to the pathogenesis of the diseases which is a completely different use of repetitive polymorphism (18).

No consensus exists in how many loci should be analyzed and how many of them should show alterations to be classified as 'high' MI. A number of publications have classified tumors as MI-positive, when as few as one of two loci appeared unstable. Other investigators have used the absence of alterations in three, four, or even five markers as the criterion for labeling the tumor specimens as 'low' MI (19). The STRs used in forensic identification, consequently in this study are not cancer markers and are situated in the noncoding regions of DNA.

The above are examples of the usage of altered microsatellites as cancer markers. However, in forensic identification the same alterations on STRs might wrongly exclude persons. Thus, we might be comparing two samples originating from the same person, but the alteration in one sample may wrongly indicate another source. In this case we have to study the risk of such a mismatch probability. Vauhkonen et al showed that the extent of the alteration for each single locus may occur at different rates for different malignancies (20). While for urinary tract malignancy we see $0 \%$ alteration, lung cancer may show up to $68 \%$. The loci themselves can also be classified as the most affected ones such as FGA and vWA and less affected ones like TH01 (21). Therefore, we have to be aware of the risk of incorrect interpretation in personal identification cases. Repeated results have shown a maximum of 5 microsatellite marker alterations in a certain battery of forensically used STRs (22). However, to exclude any doubt we wanted to include in our battery the maximum number $(n=15)$ of STRs that have been standardized as polymorphic markers.

Circulating DNA in the blood of many patients with breast and other types of cancer has been detected because of cell lysis, tumor necrosis, apoptosis, and active cell shedding (2). A high proportion of breast cancer patients exhibited plasma DNA at diagnosis similar to tumor DNA, and its presence correlated significantly with pathological parameters associated with a poor prognosis (23).

It is clear that the interpretation of DNA findings in cancer cases should be done extremely carefully because the potential for genetic instability of the STR markers is considered to be high. Therefore, in our study in order to avoid any possible misinterpretation we used the maximum number of available standardized polymorphic loci for typing the malignant tissue and we compared the findings with blood and buccal swabs. The buccal swabs were used as an unaffected histologically normal tissue sample. It was confirmed in our case that there was no mixing of any mutated plasma DNA in the blood sample that we used (Table I).

In a study where the psychological squeal of false-positive recall in women with and without a family history of breast cancer was studied, depression, stress-related behavior changes border line or clinically significant anxiety or at least shortterm distress has been detected. The breast screening programs should ensure that steps are taken to minimize the number of women who are recalled for further investigations (24), highlighted by the psychological effect that will result from an unnecessary total mastectomy, as happened to the case we have described.

In conclusion, the excisional biopsy is a valuable diagnostic tool in breast cancer but there is always a possibility of unintentional interchange of the biopsy material resulting to unpleasant consequences. DNA technology is used for human identification and defined as reliable, it can be easily applied to prove the identity of the samples. However, the interpretation should be done with extreme care because the possibility of genetic instability in tumor cells. Therefore, the maximum possible number of polymorphic markers should be analyzed. Misinterpretation and false-negatives may result in a delay in the treatment of the patient. We based our deductions on the assumption of unintentional interchange of the biopsy material. We should always have in mind that there may be cases of intentional interchange. In such cases the DNA result will form evidence for medico-legal investigations and this will change completely the way of approaching the case.

\section{References}

1. The Republic of Turkey Ministry of Health www.saglik.gov.tr.

2. Gilbey AM, Burnett D, Coleman RE and Holen I: The detection of circulating breast cancer cells in blood. J Clin Pathol 57: 903-911, 2004.

3. Kopans DB: Mammography screening is saving thousands of lives, but will it survive medical malpractice? Radiology 230: 20-24, 2004.

4. Berlin L: Mammography screening can survive malpractice ... If radiologists take center stage and assume the role of educator. Radiology 233: 641-644, 2004.

5. Nodine CF, Mello-Thoms C, Weinstein SP, Kundel HL, Conant EF, Heller-Savoy RE, Rowlings SE and Birnbaum JA: Blinded review of retrospectively visible unreported breast cancers: an eye-position analysis. Radiology 221: 122-129, 2001.

6. Kopans DB: Be careful to not willingly suspend disbelief. Radiology 233: 645-647, 2004.

7. Banaschak S, Du Chesne A and Brinkmann B: Multiple interchanging of tissue samples in cases of breast cancer. Forensic Sci Int 113: 3-7, 2000.

8. Reilly P: Physician responsibility in conducting genetic testing. J Natl Cancer Inst Monogr 17: 59-61, 1995.

9. AmpFISTR ${ }^{\circledR}$ Identifiler $^{\mathrm{TM}}$ PCR Amplification Kit User's Manual ${ }^{\odot}$ Copyright 2001, Applied Biosystems, USA.

10. Holt C, Stauffer C, Wallin J, Lazaruk L, Nguyen T, Budowle B and Walsh P: Practical applications of genotypic surveys for forensic STR testing. Forensic Sci Int 112: 91-109, 2000

11. Butler J: Forensic DNA typing. Academic Press, San Diego, CA, 2001

12. DNA Advisory Board, Federal Bureau of Investigation, U.S. Department of Justice. Quality assurance standards for forensic DNA testing laboratories. 1998.

13. Kimpton CP, Oldroyd NJ, Watson SK, Frasier RRE, Johnson PE, Millican ES, Urquhart A, Sparkes RL and Gill P: Validation of highly discriminating multiplex short tandem repeat amplification systems for human identification. Electrophoresis 17: 1283-1293, 1996. 
14. Yee CJ, Roodi N, Verrier CS and Parl FF: Microsatellite instability and loss of heterozygosity in breast cancer. Cancer Res 54: 1641-1644, 1994.

15. Kiaris H, Ergazaki M and Spandidos DA: Instability at the H-ras minisatellite is associated with the spontaneous abortion of the embryo. Biochem Biophys Res Commun 214: 788-792, 1995.

16. Vassilakis DA, Sourvinos G, Markatos M, Psathakis K, Spandidos DA, Siafakas NM and Bouros D: Microsatellite DNA instability and loss of heterozygosity in pulmonary sarcoidosis. Am J Respir Crit Care Med 160: 1729-1733, 1999.

17. Spandidos DA, Sourvinos G, Kiaris H and Tsamparlakis J: Microsatellite instability and loss of heterozygosity in human pterygia. Br J Ophthalmol 81: 493-496, 1997.

18. Paraskakis E, Sourvinos G, Passam F, Tzanakis N, Tzortzaki EG, Zervou M, Spandidos D and Siafakas NM: Microsatellite DNA instability and loss of heterozygosity in bronchial asthma. Eur Respir J 22: 951-955, 2003.

19. Bocker T, Diermann J, Friedl W, Gebert J, Holinski-Feder E, Karner-Hanusch J, von Knebel-Doeberitz M, Koelble K, Moeslein G, Schackert HK, Wirtz HC, Fishel R and Ruschoff J: Microsatellite instability analysis: a multicenter study for reliability and quality control. Cancer Res 57: 4739-4743, 1997.
20. Vauhkonen H, Hedman M, Vauhkonen M, Kataja M, Sipponen P and Sajantila A: Evaluation of gastrointestinal cancer tissues as a source of genetic information for forensic investigations by using STRs. Forensic Sci Int 139: 159-167, 2004.

21. Paia C-Y, Hsiehb L-L, Tsaic C-W, Chiouc F-S, Yangc C-H and Hsu B-D: Allelic alterations at the STR markers in the buccal tissue cells of oral cancer patients and the oral epithelial cells of healthy betel quid-chewers: an evaluation of forensic applicability. Forensic Sci Int 129: 158-167, 2002.

22. Brinkmann B, Klintschar M, Neuhuber F, Huhne J and Rolf B: Mutation rate in human microsatellites: influence of the structure and length of the tandem repeat. Am J Hum Genet 62: 1408-1415, 1998.

23. Silva JM, Dominguez G and Garcia JM: Presence of tumor DNA in plasma of breast cancer patients: clinicopathological correlations. Cancer Res 59: 3251-3256, 1999.

24. Gilbert F, Cordiner C, Affleck I, Hood D, Mathieson D and Walker L: Breast screening: the psychological sequelae of falsepositive recall in women with and without a family history of breast cancer. Eur J Cancer 34: 2010-2014, 1998. 